\title{
Image formation and data acquisition in a stage scanning 4Pi confocal fluorescence microscope
}

\author{
Juhani T. Soini, Martin Schrader, Pekka E. Hänninen, and Stefan W. Hell
}

\begin{abstract}
We describe the three-dimensional (3-D) image formation and data acquisition in a stage scanning 4Pi confocal fluorescence microscope with the use of two-photon excitation. The 3-D point-spread functions of the $4 \mathrm{Pi}$ confocal and regular confocal microscope are measured and compared. Particular emphasis is given to the data acquisition procedure. 4Pi confocal microscopy results in a point-spread function that is 4 times sharper than that of a regular confocal microscope, ultimately leading to superior 3-D imaging of translucent fluorescent specimens. For a two-photon excitation wavelength of approximately $800 \mathrm{~nm}$, we obtain an axial resolution of $140 \mathrm{~nm}$. (c) 1997 Optical Society of America
\end{abstract}

\section{Introduction}

Confocal microscopy is an expanding area of research with various applications in medical and biological sciences. $^{1-4}$ The need for higher resolution in farfield light microscopy has spurred new research activities and has led to new approaches. ${ }^{5-12} 4 \mathrm{Pi}$ microscopy has been described as an approach with fundamentally increased axial resolution. ${ }^{6,13}$ It has been shown that this improved resolution leads to a fourfold to fivefold improved axial sectioning. ${ }^{14,15}$ As a consequence, it is possible to perform thinner axial slices. The improvement of axial resolution has been well quantified by the measurement of the point-spread function (PSF) of 4Pi confocal microscopes by means of scattering subresolution particles. From the measured PSF the optical transfer function was derived. ${ }^{16}$ The measured PSF was found in a good agreement with theoretical predictions. The measurements are also relevant for single-photon excitation fluorescence microscopy. Recently a promising approach has been reported that combines $4 \mathrm{Pi}$ confocal microscopy with a phase-conjugate mirror. ${ }^{17}$

We describe the image and data acquisition procedure in a $4 \mathrm{Pi}$ microscope system with an object-

The authors are with the Department of Medical Physics and Chemistry, University of Turku, FIN-20521 Turku, Finland. M. Schrader and S. W. Hell are also with the High Resolution Optical Microscopy Group, Max-Planck-Institute for Biophysical Chemistry, D-37070 Göttingen, Germany. E-mail: SHell@gwdg.de.

Received 31 March 1997; revised manuscript received 11 August 1997.

0003-6935/97/348929-06 $\$ 10.00 / 0$

(C) 1997 Optical Society of America scanning piezoelectric setup of high precision. We measure the PSF of the two-photon version of a $4 \mathrm{Pi}$ confocal microscope and compare it with the confocal counterpart. We demonstrate the fourfold increased axial resolution by imaging densely clustered fluorescent beads that cannot be resolved by use of a standard confocal microscope.

\section{Theory}

The theoretical foundations of confocal and 4Pi confocal microscopy are best derived from the amplitude PSF of a lens that, in the scalar approximation, is given by

$$
h(u, v)=c \int_{0}^{1} P(\rho)\left(\exp \left(-\frac{1}{2} i u \rho^{2}\right) J_{0}(v \rho) \rho \mathrm{d} \rho,\right.
$$

with $c$ being a constant, $P(\rho)$ the pupil function, and $J_{0}$ a zero-order Bessel function of the first kind.

$$
v=(2 \pi / \lambda) n r \sin \alpha, \quad u=(4 \pi / \lambda) n z \sin ^{2}(\alpha / 2)
$$

are optical coordinates. ${ }^{18}$ In a confocal microscope we have two consecutive imaging steps, the first step being the illumination and the second the detection. Therefore Eq. (1) becomes

$$
h_{\mathrm{conf}}=h_{\mathrm{ill}}(u, v) \times h_{\mathrm{det}}(u / \beta, v / \beta),
$$

where $\beta=\lambda_{\text {det }} / \lambda_{\text {ill }}$ is the ratio of the illumination and the fluorescence wavelengths. ${ }^{1,2,4}$ The modulussquared confocal amplitude PSF describes the probability of each focal coordinate to contribute to the fluorescence signal. The spatial extent of the intensity PSF is a good measure of the resolution. High- 


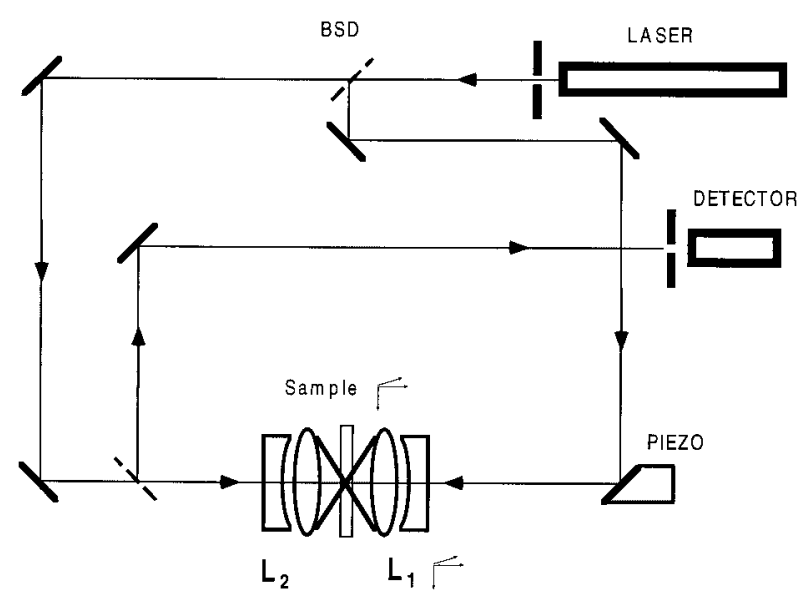

Fig. 1. 4Pi-confocal microscope. Laser light coming from a pinhole is split into two parts by a beam splitter (BSD), thus illuminating simultaneously two opposing high-aperture lenses. The light originating from the sample is collected by the lens $\mathrm{L}_{2}$ and directed toward a detector pinhole. The relative phase of the illumination wave fronts is adjusted by means of a piezoelectrically driven mirror. The lens $\mathrm{L}_{2}$ is fixed and the lens $\mathrm{L}_{1}$ is adjusted with a precision of $10-20 \mathrm{~nm}$ with respect to $L_{2}$. The sample is scanned with a piezoelectric stage of high precision to render a 3-D image.

resolution microscopy aims to reduce the threedimensional (3-D) extent of the PSF.

In a $4 \mathrm{Pi}$ confocal microscope of type $\mathrm{A}$, the axial resolution is increased by use of two coherently illuminated objective lenses with a common focus (Fig. 1). The $4 \mathrm{Pi}$ amplitude illumination PSF is the sum of the two counterpropagating wavefronts of the objective lenses $L_{1}$ and $L_{2}$. In the case of two similar objectives we obtain

$$
h_{\mathrm{ill}, 4 \mathrm{Pi}}=h_{1, \mathrm{ill}}(u, v)+h_{2, \mathrm{ill}}(-u, v) .
$$

Figure 2 depicts the $4 \mathrm{Pi}$ illumination intensity PSF and the single-lens counterpart for a N.A. of 1.4, $\lambda=$ $500 \mathrm{~nm}$, and oil immersion $(n=1.518)$ for constructive interference. Coherent illumination through both lenses renders a PSF that has a maximum that is approximately 4 times narrower in the axial direction than its single-lens counterpart. The 4Pi illumination intensity PSF also features two pronounced axial lobes, but the narrower main maximum opens up the prospect of increased axial resolution. ${ }^{6}$ The lobes stem from the fact that the resulting wave front produced by the two objective lenses does not reach the full solid angle of $4 \pi$. There are different ways to suppress the lobes. First, confocal detection reduces the lobes in the resulting PSF because of the suppression of out-of-focus contribution. Second, the excitation of the fluorophore can be performed with two-photon excitation (TPE), which reduces fluorescence in the outer regions of the focus owing to the nonlinear dependence on the excitation intensity. ${ }^{6}$ Third, we can remove the lobes by assuming that the axial profile of the $4 \mathrm{Pi}$ confocal PSF is a convolution of two functions: the lobe function, $l(r$, $z$ ), and the peak intensity function, $\left|h_{\text {peak }}(r, z)\right|^{2}$ (also shown in Fig. 3). As a good approximation, the lobe function is the sum of (three) offset $\delta$ functions weighted by the height of the ordinary TPE confocal $\mathrm{PSF}$, describing the location of the lobes and their relative heights. ${ }^{15}$ The peak function quantifies the intensity distribution of a single peak as it is encountered in the axial profile of the 4Pi PSF:

$$
\left|h^{4 \mathrm{Pi}}(r, z)\right|^{2}=l\left(r, z^{\prime}\right) \otimes_{z^{\prime}}\left|h_{\text {peak }}\left(r, z^{\prime}\right)\right|^{2} .
$$

In the Fourier space, this becomes $H^{4 \mathrm{Pi}}=L H_{\text {peak }}$. The lobe function, and thus the lobes, can be computationally removed by multiplying the $4 \mathrm{Pi}$ response by the inverse of the lobe function in the Fourier space. The resulting response is a single peak. It is practical to carry out the inversion of the lobe function in the spatial domain. With $l^{-1}\left(z^{\prime}\right)$ denoting the inverse Fourier transform of $1 / L$, we obtain

$$
\left|h_{\text {peak }}(r, z)\right|^{2} \approx\left|h^{4 \mathrm{Pi}}\left(r, z^{\prime}\right)\right|^{2} \otimes_{z^{\prime}} l^{-1}\left(z^{\prime}\right) .
$$

For an object $O(r, z)$, the deconvolved image from a $4 \mathrm{Pi}$ microscope is described by

$$
\begin{aligned}
I(r, z) & =\left|h_{\text {peak }}\left(r, z^{\prime}\right)\right|^{2} \otimes_{z^{\prime}} O\left(r, z^{\prime}\right) \\
& =\left[\left|h^{4 \mathrm{Pi}}\left(r, z^{\prime \prime}\right)\right|^{2} \otimes_{z^{\prime \prime}} O\left(r, z^{\prime \prime}\right)\right] \otimes_{z^{\prime}} l^{-1}\left(r, z^{\prime}\right) .
\end{aligned}
$$

This filter performs well when the lobes are below $50 \%$, which makes it a good combination with TPE 4Pi confocal microscopy. The fact that the filter consists of points allows for a rapid on-line application of the deconvolution shortly after image recording. For lobes at approximately $50 \%$ or higher, five and more points are required for deconvolution. The information needed for performing such a point deconvolution is the distance between the lobes and their relative heights. The point deconvolution must not be confused with extensive PSF deconvolution procedures that require more extensive computations. ${ }^{19}$ Fig. $3 \mathrm{~d}$ is actually the result achieved by the application of the three-point inverse filtering to the measurement of the 4Pi intensity PSF of Fig. 3b. After deconvolution, the axial resolution of the $4 \mathrm{Pi}$ confocal microscope is described by Fig. 3d. A comparison between Fig. 3d and its single-lens counterpart (Fig. 3a) shows an experimental axial resolution improvement of approximately 4 in the 4Pi confocal microscope. We later apply the three-point deconvolution to 3-D image stacks.

\section{Data Acquisition in 4Pi Confocal Setup}

In the setup of Fig. 1, the lenses $L_{1}$ and $L_{2}$ face each other, with $\mathrm{L}_{2}$ fixed in its position and $\mathrm{L}_{1}$ mounted on a piezoelectrical stage (Melles Griot, Cambridge, UK). The stage allows for an accurate positioning $(10 \mathrm{~nm})$ of the second objective with respect to the first one to ensure the overlap of their illumination foci. The object is illuminated through both objective lenses with the expanded beam of a mode-locked Ti:sapphire laser that is divided into two equally intense beams by the beam splitter BSD. The object is mounted on a similar stage and is scanned through the common focus of both objectives, and the fluores- 


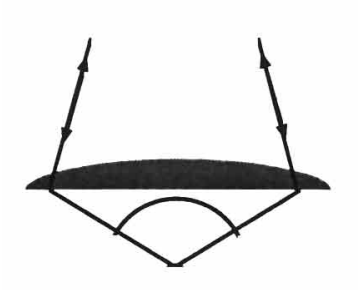

Intensity PSF
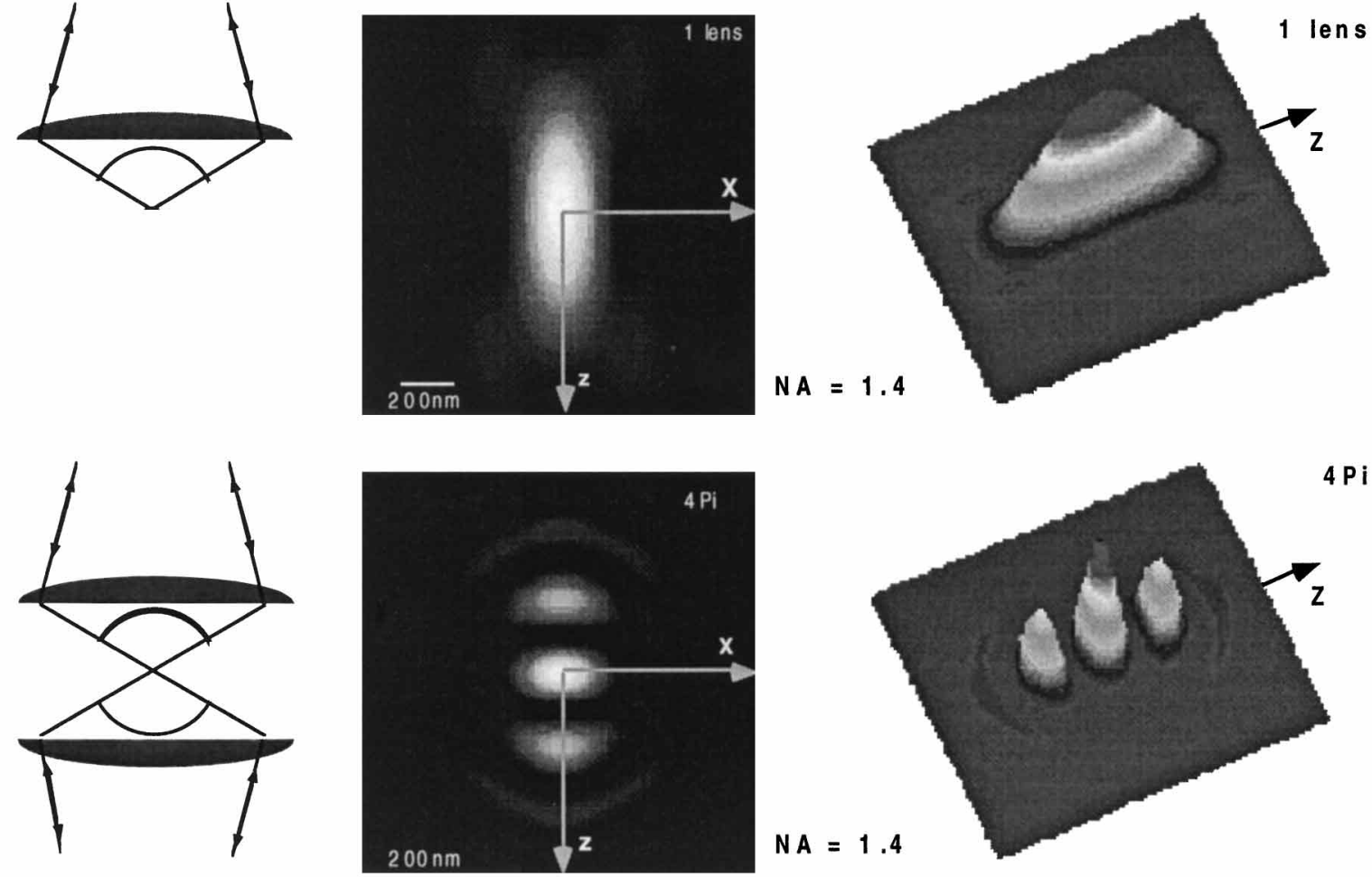

Fig. 2. Calculated PSF of a single lens (above) compared with calculated PSF of a 4Pi arrangement (below). 4Pi microscopy makes use of two opposing objective lenses of high numerical aperture (here N.A. $=1.4$ oil and $\lambda=500 \mathrm{~nm}$ ) with a common focus. The coherent addition of the electric fields yields a steep main maximum and two lobes in the axial direction. The center panels outline the focal intensities in a plane that contains the optical axis, whereas the right-hand panels show the corresponding surface plots. As with the single lens PSF's, the 4Pi PSF gives the relative probability that a photon from a pinhole arrives at a certain point in the focal region when propagated simultaneously through both lenses. The 4Pi PSF is shown for constructive interference of the illuminating waves at the focal point.

cence light is collected by $\mathrm{L}_{2}$ and focused upon a pinhole in front of the detector $\mathrm{D}$.

Figure 4 shows the scheme of the data acquisition and detection system. During data acquisition the sample is scanned continuously at approximately 2 $\mathrm{Hz}$ in the axial direction, performing line scans. The movement of the first axis is called fast scanning, and the direction of this movement is referred to as the fast axis. The function generator that provides the input for the controller of the scan stages also sends a trigger signal to a multi-input-output I/O card. The LABVIEW-based control software governs the movement of the second and third scan axis through this I/O card. After completion of each line scan, indicated by the trigger signal, the second scan axis is moved toward the starting point for the next line. Likewise, after completion of the preset number of line scans, the stage is moved in the direction of the third axis for starting to generate another image, which is laterally displaced to the previous one.

In $4 \mathrm{Pi}$ confocal microscopy, the fast axis is preferrably the optical axis $(z)$. There are two reasons for this. First, the initial alignment of the lenses and the adjustment of the relative phase of the two wave fronts are best accomplished through monitoring the axial scan. Second, the $4 \mathrm{Pi}$ confocal microscope pro- vides $20-25 \%$ better resolution in the axial direction than in the lateral direction so that it is useful to have denser pixelation in the optical axis. Therefore our $x-z$ images represent slices of 3 -D stacks of $x-z$ images, with the $z$ axis being the fast-scan axis.

The trigger signal provided by the function generator is also used as the start-of-sweep signal for the multichannel scalar card, which collects the transistortransistor logic pulses of the avalanche diode singlephoton counting module (EG\&G Model SPCM-131). The transistor-transistor logic pulses represent single photons. Photon counting with an avalanche photodiode is useful for increasing the efficiency of detection of low signals, especially in the red regime where the diode features quantum efficiencies of detection of 60-70\%. The choice of the channel dwell time of the multichannel scalar card in combination with the scan speed of the stage determines the axial pixel size in the recorded 3-D data set. In our experiments the scan speed was approximately 10 $\mu \mathrm{m} / \mathrm{s}$. The dwell time was approximately $2 \mathrm{~ms}$. Thus the axial pixel size was approximately $20 \mathrm{~nm}$, which is well above the density required by the Nyquist criterion.

The LABVIEW program and the I/O card control the movement of the stages as well as the data acquisi- 

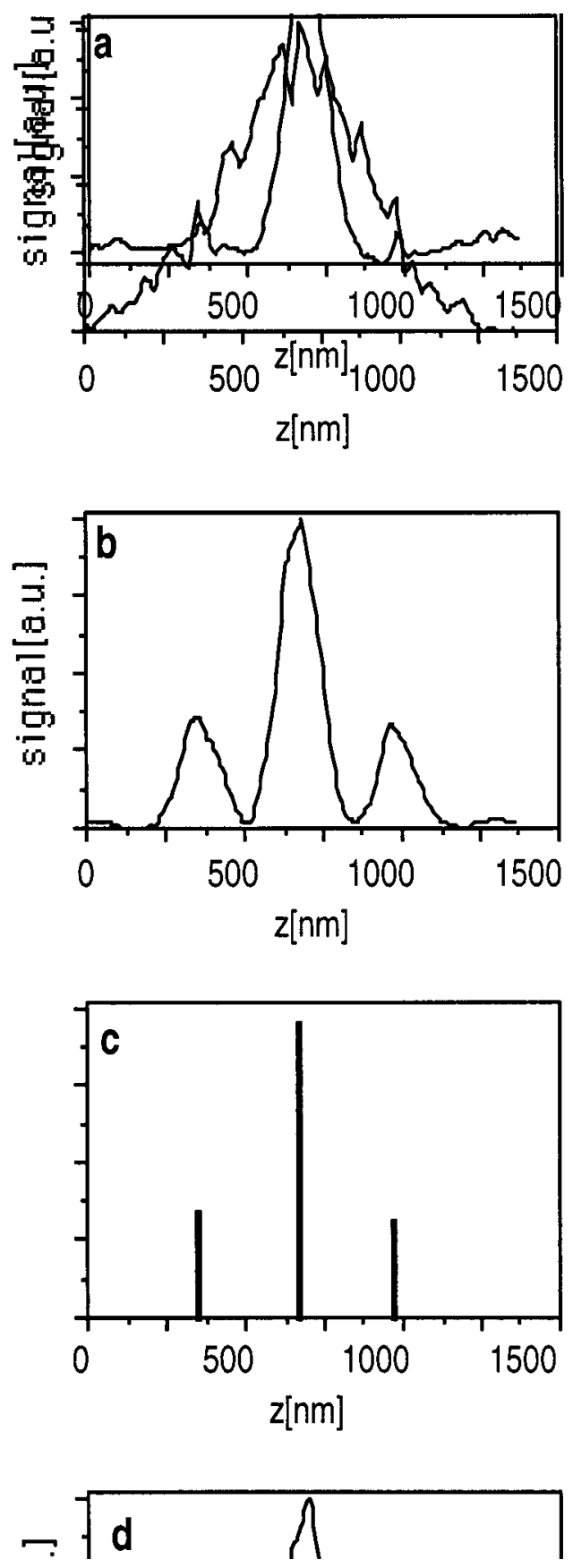

Fig. 3. Axial responses through the bead images (PSF's) of Fig. 5: a, confocal; b, 4Pi confocal; c, lobe function $l(r, z)$; and $\mathrm{d}$, peak function $h_{\text {peak }}$. The peak function quantifies the axial resolution of a TPE 4Pi confocal microscope with deconvolution by the inverse of $l(r, z)$. A comparison of view d with view a shows a fourfold increase of the axial resolution in $4 \mathrm{Pi}$ confocal microscopy.

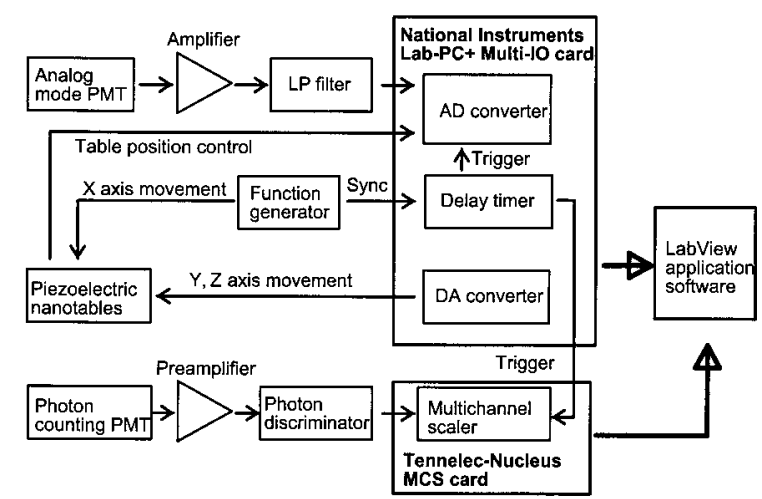

Fig. 4. Block diagram of control and data acquisition system for 4Pi microscope.

tion. The feedback signal of the piezos can be used to monitor precisely the actual position of the stages and to map the recorded intensity data accordingly. In our experiments we used a sawtooth ramp to drive the fast axis movement of the stage. Thus the axial movement was linear, and no further data manipu-
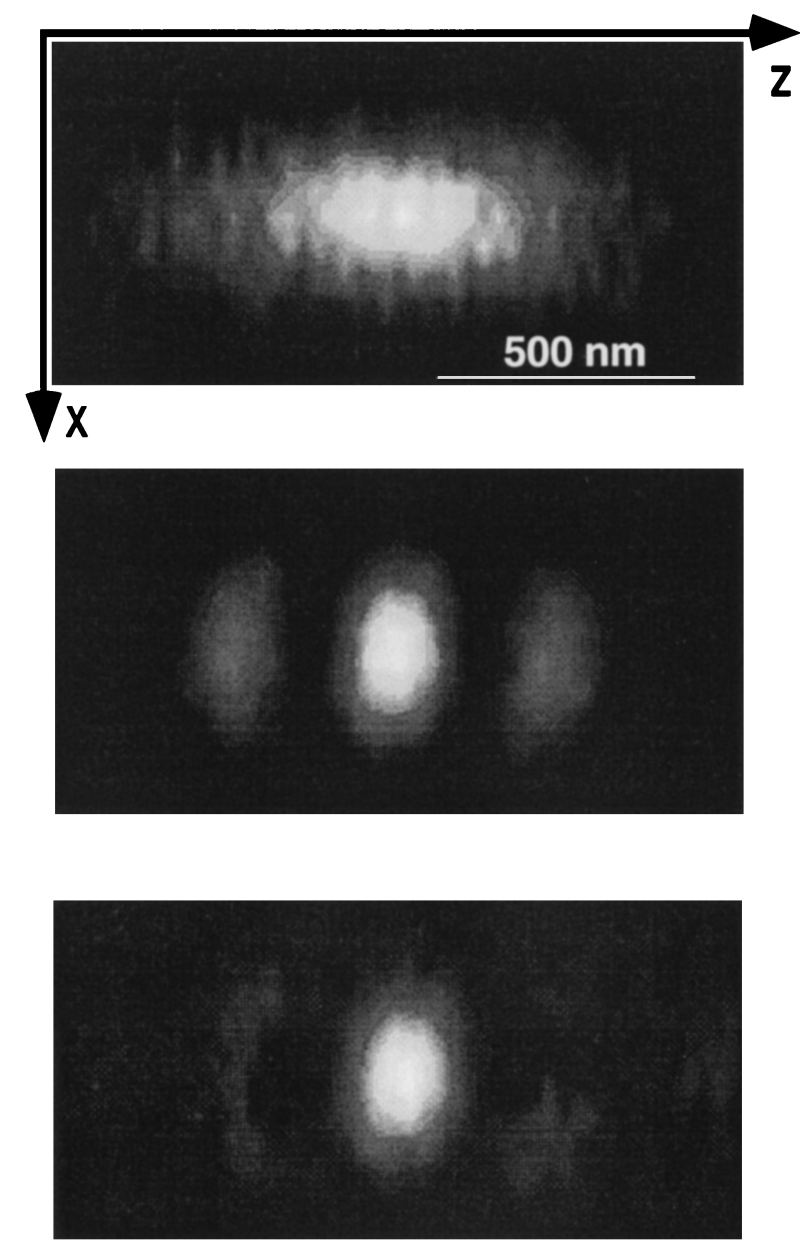

Fig. 5. Axial image of 115-nm bead, which, as a good approximation, corresponds to (top) PSF of the confocal microscope, (middle) $4 \mathrm{Pi}$ confocal microscope, and (bottom) 4Pi confocal microscope after inverse point filtering. 

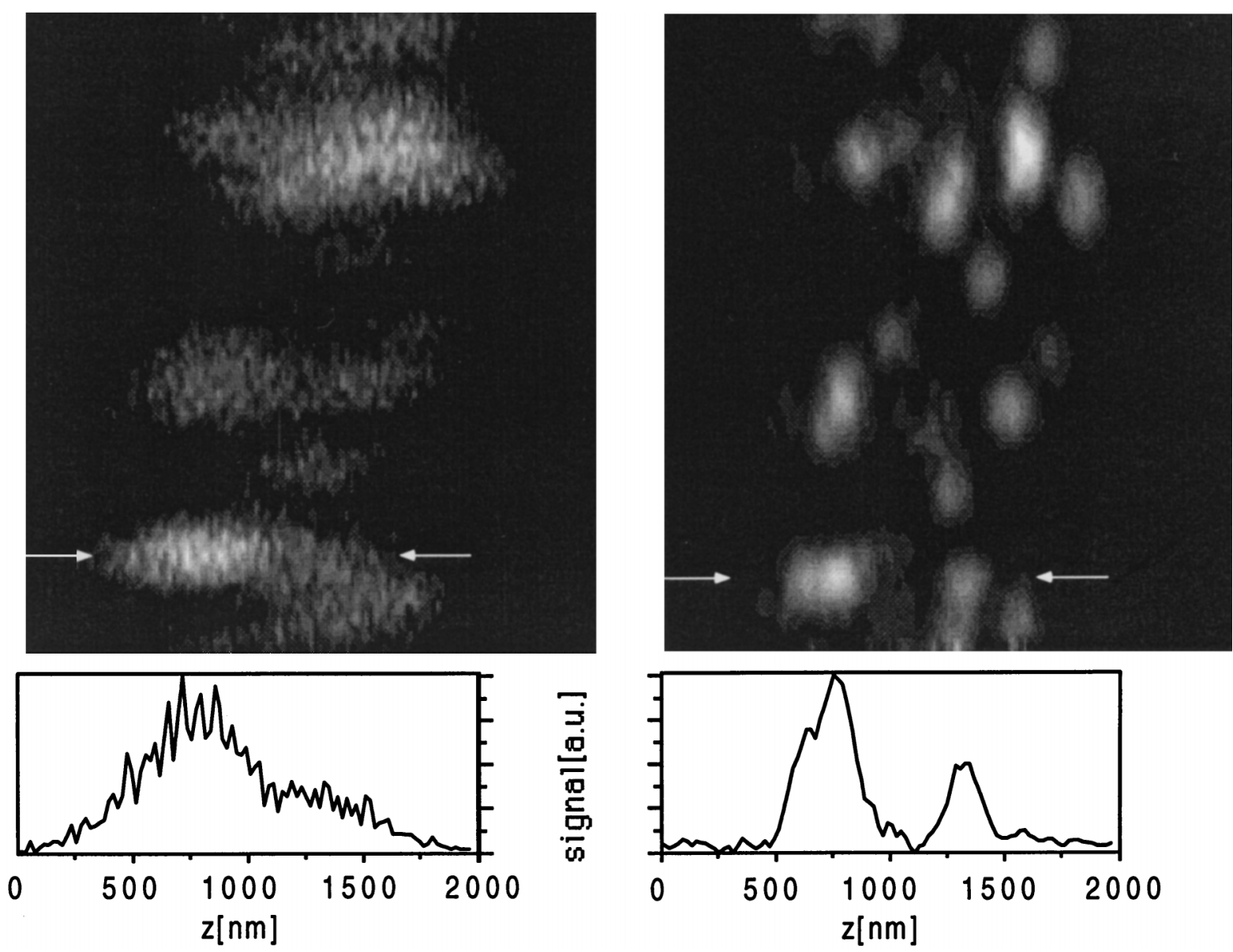

Fig. 6. Axial slice through 3-D data stack of clustered beads as performed with confocal microscope (left-hand panel) and 4Pi confocal microscope (right-hand panel). The intensity profiles are performed along the marked line in the image. The 4Pi confocal image of the same object is fundamentally superior in signal and in resolution.

lation was needed to correct for a nonlinear ramp. The stored 3-D data stacks can be imported into any imaging software that allows for data display and processing. We preferred the public domain program NIH ImAGE (National Institutes of Health, Bethesda, Maryland). The point deconvolution of the 4Pi images was implemented as a macro in the program and performed after data acquisition. In principle, point deconvolution can also be performed on-line.

\section{Imaging in 4Pi Confocal Microscope}

Fig. 5 shows a comparison of the various PSF's: (top) confocal, (middle) 4Pi-confocal and (bottom) 4Piconfocal after three-point inverse filtering in the $x-z$ plane that contains the optical axis. As a test object we used a latex bead approximately $115 \mathrm{~nm}$ in diameter, labeled with a red fluorescent fluorophore. The bead was two-photon excited by the Ti:sapphire laser at a wavelength of $815 \mathrm{~nm}$. Strictly speaking, Fig. 5 shows bead images instead of the pure PSF. However, the bead diameter of approximately $115 \mathrm{~nm}$ is comparatively small. Moreover, the FWHM of the dye distribution in the object function of the bead can be calculated to be $80 \mathrm{~nm}$. Hence one can infer that, to a good approximation, the bead images show the
PSF of the corresponding microscopes. Figure 5 shows single slices of 3-D data stacks that contain 20 axial images that are $50 \mathrm{~nm}$ apart in the $y$ direction. The pixel sizes in the $x$ and the $z$ directions are 54 and $27 \mathrm{~nm}$, respectively, so that the axial pixelation is approximately twice as dense as in the lateral direction. The intensity profiles on the right-hand side of the images reveal the reduced axial extent of the main maximum in the 4Pi PSF compared with the confocal PSF. The axial FWHM in the confocal image is approximately $580 \mathrm{~nm}$ and in the $4 \mathrm{Pi} \mathrm{PSF}$ approximately $140 \mathrm{~nm}$. In the bottom view of Fig. 5 the effective PSF after three-point inverse filtering is shown, exhibiting the same axial FWHM as in the middle view. The lateral FWHM, which is approximately $200 \mathrm{~nm}$ in this experiment, is not altered in the 4Pi microscope. It is interesting that the effective PSF in the bottom view of Fig. 5 is sharper in the axial than in the lateral direction and, besides improving the resolution, also promises a higher image fidelity.

Figure 6 demonstrates the improvement of resolution in the imaging of densely clustered fluorescent beads. In the confocal image the elongation along the optical axis makes the separation of the bead clusters 
impossible. In contrast, the 4Pi confocal microscope, benefiting from the increased axial resolution, resolves the beads in the clustered regions of the image. This is the case throughout the 3-D data stack.

The data profiles of Fig. 6 quantify the improvement of the axial resolution: The confocal image shows a very elongated profile so that individual beads cannot be distinguished. The 4Pi image separates the beads at $z=1300 \mathrm{~nm}$ from the signal at $z$ $=750 \mathrm{~nm}$ and also separates two beads within this peak at $z=750 \mathrm{~nm}$ at a distance of approximately $120 \mathrm{~nm}$. One can recognize two beads (of $115 \mathrm{~nm}$ diameter) that are in direct contact with each other. Thus we demonstrate a microscope suitable for 3-D imaging of fluorescent objects with an axial resolution that surpasses the resolution of confocal microscopes by a factor of 4 .

Finally we note that the confocal and the $4 \mathrm{Pi}$ confocal images were generated with the average power of approximately $300 \mu \mathrm{W}$. The $4 \mathrm{Pi}$ images exhibit a better signal-to-noise ratio, though. This is attributed to the higher local intensity in the 4Pi PSF, resulting from the interference of the two wave fronts in conjunction with the quadratic dependence of excitation on intensity in two-photon fluorescence microscopy.

\section{Conclusion}

We have described the image formation and the data acquisition in a stage scanning $4 \mathrm{Pi}$ confocal microscope with use of TPE fluorescence. We have determined the 3-D PSF of a two-photon confocal microscope and a two-photon 4Pi confocal microscope and have shown that the latter is suitable for improving the axial resolution and 3-D imaging fidelity as a whole. This improvement is due to a more spherical effective spot in 4Pi confocal microscopy, which is obtained after fast (three-point) deconvolution. The improved resolution and imaging fidelity resulted in fundamentally superior 3-D images of clustered subresolution fluorescence beads compared with regular confocal image, thus accounting for what is, to our knowledge, the sharpest 3-D focus with light at present. However, it is clear that the resolution of $4 \mathrm{Pi}$ confocal microscopy can be improved further by a deconvolution with the 4Pi PSF. ${ }^{20,21}$ Indeed, at a TPE wavelength of $810 \mathrm{~nm}$, an unprecedented 3-D resolution in the $100-\mathrm{nm}$ range in fibroblast cells has been shown. ${ }^{21}$

We thank Alex Egner for carefully reading the manuscript. This work was carried out in the framework of a research agreement between S. W. Hell and EG\&G Wallac Oy. Most of the work reported here was carried out while M. Schrader and S. W. Hell were recipients of a grant by the European Commission.

\section{References}

1. T. Wilson and C. J. R. Sheppard, Theory and Practice of Scanning Optical Microscopy (Academic, New York, 1984).
2. T. Wilson, Confocal Microscopy (Academic, London, 1990).

3. J. Pawley, Handbook of Biological Confocal Microscopy (Plenum, New York, 1995).

4. M. Gu, Principles of Three-Dimensional Imaging in Confocal Microscopes (World Scientific, Singapore, 1996).

5. M. Bertero, P. Boccacci, G. J. Brakenhoff, F. Malfanti, and H. T. M. van der Voort, "Three-dimensional image restoration and super-resolution in fluorescence confocal microscopy," J. Microsc. 157, 3-20 (1990).

6. S. Hell and E. H. K. Stelzer, "Fundamental improvement of resolution with a $4 \mathrm{Pi}$-confocal fluorescence microscope using two-photon excitation," Opt. Commun. 93, 277-282 (1992).

7. B. Bailey, D. L. Farkas, D. L. Taylor, and F. Lanni, "Enhancement of axial resolution in fluorescence microscopy by standing-wave excitation," Nature (London) 366, 44-48 (1993).

8. S. W. Hell, "Improvement of lateral resolution in far-field light microscopy using two-photon excitation with offset beams," Opt. Commun. 106, 19-22 (1994).

9. W. A. Carrington, R. M. Lynch, E. D. W. Moore, G. Isenberg, K. E. Fogarty, and F. S. Fay, "Superresolution in threedimensional images of fluorescence in cells with minimal light exposure," Science 268, 1483-1487 (1995).

10. H. Kano, H. T. M. van der Voort, M. Schrader, G. van Kempen, and S. W. Hell, "Avalanche photodiode detection with object scanning and image restoration provides 2-4 fold resolution increase in two-photon fluorescence microscopy," Bioimaging 4, 187-197 (1996).

11. M. Müller and G. J. Brakenhoff, "Using offset interfering beams for improved resolution in confocal imaging: the potential of the PSAF-technique," Bioimaging 4, 179-186 (1996).

12. M. Vaez-Iravani and D. I. Kavaldjiev, "Resolution beyond the diffraction limit using frequency-domain field confinement in scanning microscopy," Ultramicrosc. 61, 105-110 (1995).

13. M. Gu and C. J. R. Sheppard, "Three-dimensional transfer functions in 4Pi confocal microscopes," J. Opt. Soc. Am. A 11, 1619-1627 (1994).

14. M. Schrader and S. W. Hell, "4Pi-confocal images with axial superresolution,” J. Microsc. 183, 189-193 (1996).

15. P. E. Hänninen, S. W. Hell, J. Salo, E. Soini, and C. Cremer, "2-photon excitation 4pi confocal microscope-enhanced axial resolution microscope for biological research," Appl. Phys. Lett. 66, 1698-1700 (1995).

16. M. Schrader, M. Kozubek, S. W. Hell, and T. Wilson, "Optical transfer functions of $4 \mathrm{Pi}$ confocal microscopes: theory and experiment," Opt. Lett. 22, 436-438 (1997).

17. Y. Kawata, K. Fujita, O. Nakamura, and S. Kawata, "4Pi confocal optical system with phase conjugation," Opt. Lett. 21, 1415-1417 (1996).

18. C. J. R. Sheppard and H. J. Matthews, "Imaging in highaperture optical systems," J. Opt. Soc. Am. A 4, 1354-1360 (1987).

19. P. J. Shaw, "Comparison of wide-field/deconvolution and confocal microscopy for 3D imaging," in Handbook of Biological Confocal Microscopy, J. Pawley, ed. (Plenum, New York, 1995), pp. 373-385.

20. M. Schrader, S. W. Hell, and H. T. M. van der Voort, "Potential of confocal microscopes to resolve in the 50-100 nm range," Appl. Phys. Lett. 69, 3644-3646 (1996).

21. S. W. Hell, M. Schrader, and H. T. M. van der Voort, "Far-field fluorescence microscopy with resolution in the 100-nm range," J. Microsc. 187, 1-5 (1997). 